\title{
Team Mobile Robots Based Intelligent Security System
}

\author{
Song Hiang Chia ${ }^{1}$, Jr Hung Guo ${ }^{1}$, Bo Yi $\mathrm{Li}^{1}$, Kuo Lan Su${ }^{2}$ \\ ${ }^{1}$ Graduate School of Engineering Science and Technology, National Yunlin University of Science \& Technology, Douliou, Yunlin, \\ Taiwan \\ ${ }^{2}$ Department of Electrical Engineering, National Yunlin University of Science \& Technology Douliou, Yunlin, Taiwan
}

Received: 17 Dec. 2012, Revised: 21 Feb. 2013, Accepted: 23 Feb. 2013

Published online: 1 Jun. 2013

\begin{abstract}
The article develops a team mobile robots based intelligent security system being applied in home automation. The detection modules of the system include active security modules and passive security modules. The control unit of all security modules (active security modules and passive security modules) is HOLTEK microchip. The paper focuses on the active security modules (mobile robots). Mobile robots are classified more and more teams according to the detection functions. Each team robot system includes many mobile robots to execute the same event detection. Each mobile robot has two variety interfaces, uses voice module to alarm users for event condition, and transmits the real-time event signals to the supervised computer and the other mobile robots via wireless RF interface. If the event occurs, the mobile robot can transmit the relation location (floor number) of the event to the supervised computer. The supervised computer decides the location of the event according the feedback signals from mobile robots, controls the other mobile robots moving to the event location, and recognizes the final decision output using Dempster-Shafter Evidence Theory. The belief value is over the threshold value and displays detection and decision output values on the monitor of the user interface. Finally, we present some experimental results using the team mobile robots for the fire detection and gas leakage detection on the experimental platform of the intelligent security system.
\end{abstract}

Keywords: Team mobile robots, home automation, HOLTEK microchip, wireless RF interface, Dempster-Shafter Evidence Theory

\section{Introduction}

Intelligent buildings and home can provide safety, convenience and welfare for human living in the 21 st century. The most important issue of the intelligent building is security system. In generally, the security system contains supervised system, active security modules, passive security modules and appliance control modules, uses redundant and complementally information fusion algorithms to enhance system reliability and certainty, and constructs the safety network using multiple level protections. Related security modules are fixed and passive; the mobile robot is an active system and has more flexible than fixed passive security module using in home safety guarding field.

In the past literatures, many experts research in the security system. Azegami and Fujiyoshi [1] described a systematic approach to intelligent building design. Kujuro and Yasuda [2] discussed the systems evolution in intelligent buildings. The quality of building services can be enhanced by updated information processing and communications functions of building automation systems. Finley et al. [3] presented a survey of intelligent building and reviews issues such as system perspective, subsystem services, and multi-tenant building. Chung and $\mathrm{Fu}$ expect to set up the standard of appliances and communication protocols and propose a complete system architecture with integrate control kernel to construct an intelligent building system [4].

In recent years, mobile robots have been widely applied in the security system as the active security modules. Recently more and more researches take interests in the field especially intelligent service robot. Yoichi Shimosasa et al. developed autonomous guard robot $[5,6]$ which integrated the security and service system. The robot can guide visitors in daytime and patrol at the night. There are more merits in the mobile robots to use team robot cooperation capabilities to such a large fleet of robots. In general, the control structure of the large fleet team mobile robots is classified centralized control and decentralized control. A centralized control requires robust and permanent communication

* Corresponding author e-mail: sukl@yuntech.edu.tw 
capabilities between all the robots and supervised system. A decentralized control only requires local communication between robots and supervised system [7]. The paper combines the centralized control and decentralized control in the intelligent security system.

\section{System Architecture}

The system architecture of the team mobile robot based intelligent security system is shown in Fig. 2.1. The system contains three levels. There are a passive detection level, an active detection level and a system supervised level [8]. The active detection level includes multiple team robot system. We can define each mobile robot of the team robot system \#1 to carry flame sensor to decide fire event. Each robot of the team robot system \#2 carries gas sensor to decide gas leakage in the environment, too. The system supervised level receives detection signals from active security modules and passive security modules via wireless RF interface and controls each robot of the team mobile robot system moving to the event location via wireless RF interface. Mobile robots of the team robot system use the Dempster-Shafter evidence theory to decide if the event is true or not. These modules of the intelligent security system have been finished, shown in Fig. 2.2, and are arranged in the experimental platform, shown in Fig. 2.3. The platform has three floors and contains three rooms for each floor. Mobile robots of each team robot system move in the experimental platform to detect event.

In the architecture, there are many active and passive security detection modules and appliance control modules in the intelligent security system. They are independent and autonomous and can work concurrently. Each module of the intelligent security system transmits the measurement values, parameter values and decision results to the team robot system and the supervised computer via wireless RF interface, and speaks Chinese language on real-time event status using voice module. The controller of the security modules is HOLTEK microchip. The wire security modules and wire appliance control modules transmits the signals to the supervised computer via RS485 interface. We arrange an ID code in each active security modules and passive security modules, and identify the module function by the ID code.

The user interface of the intelligent security system is shown in Fig. 2.4 for each team robot system. The user interface contains four parts. There are fire detection function, gas leakage detection function, intruder detection function and temperature detection function. Each detection function has three mobile robots. The bottom displays the detection and decision results from team robot system. We use the green label to present no event status of the environment and red label to present event status for event detection by mobile robots.

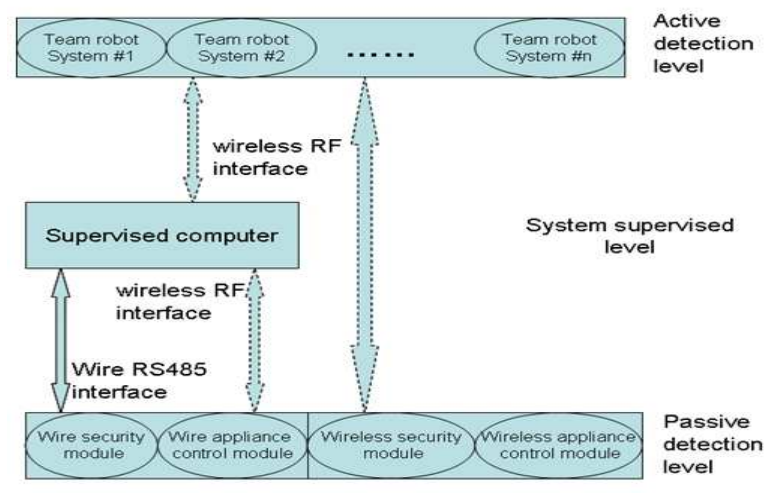

Fig. 2.1: Architecture of the Security System

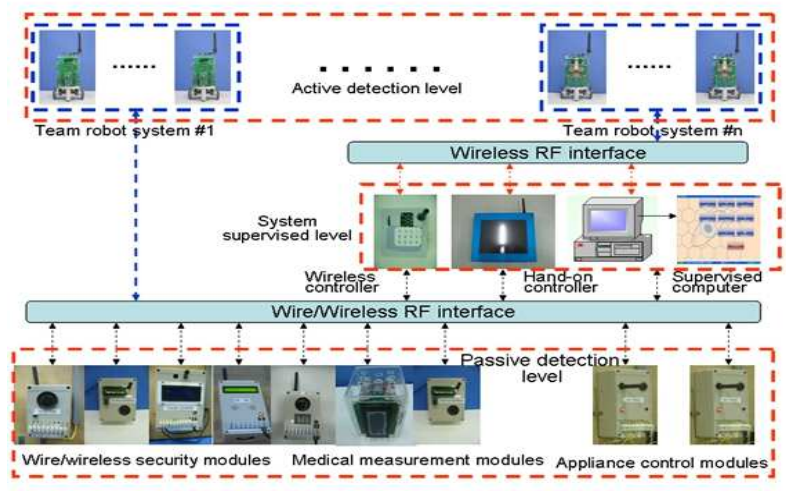

Fig. 2.2: Modules of the Security System

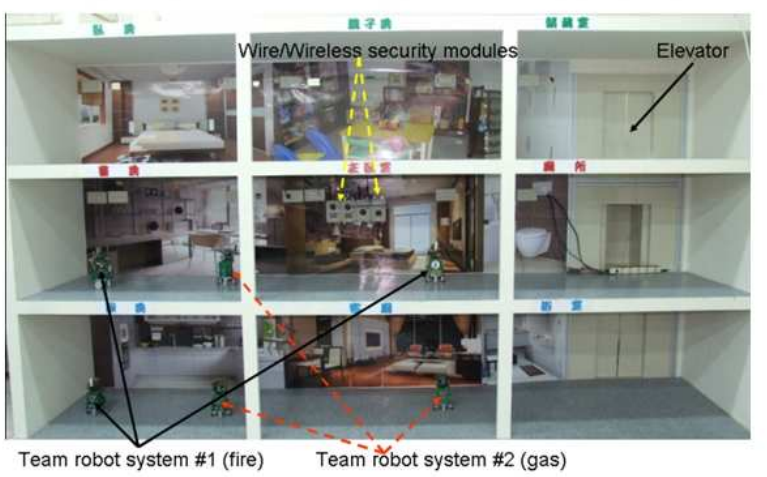

Fig. 2.3: Experimental Platform of the Security System

\section{Team Mobile Robot Systems}

The mobile robot has the shape of cylinder, and it's equipped with a microchip (HT46RU25) as controller. In the input signals, the pulse signals of the DC servomotor transmit to the controller and calculate the displacement of the mobile robots moving on the experimental 


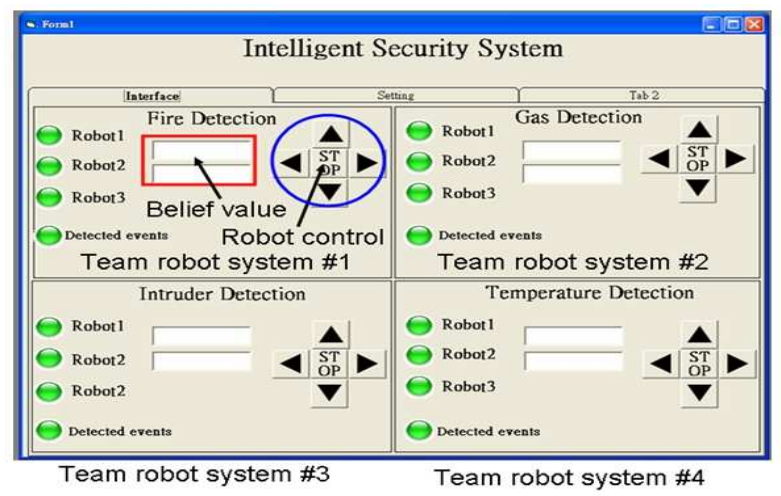

Fig. 2.4: User Interface of the Security System
Table 3.2: Command Protocol of the Supervised Computer

\begin{tabular}{|l|l|l|l|l|}
\hline $\begin{array}{l}\text { Byte } \\
\text { number }\end{array}$ & 1 & 2 & 3 & 4 \\
\hline Definition & ID code & $\begin{array}{l}\text { Robot ID } \\
\text { code }\end{array}$ & $\begin{array}{l}\text { floor } \\
\text { number }\end{array}$ & $\begin{array}{l}\text { No } \\
\text { use }\end{array}$ \\
\hline 5 & 6 & 7 & 8 & \\
\hline Target floor & No use & Orientation & No use \\
\hline
\end{tabular}

Table 3.3: Communication Protocol of the Mobile Robot

\begin{tabular}{|l|l|l|l|l|}
\hline $\begin{array}{l}\text { Byte } \\
\text { number }\end{array}$ & 1 & 2 & 3 & 4 \\
\hline Definition & ID code & $\begin{array}{l}\text { Robot ID } \\
\text { code }\end{array}$ & $\begin{array}{l}\text { Floor } \\
\text { number }\end{array}$ & $\begin{array}{l}\text { No } \\
\text { use }\end{array}$ \\
\hline 5 & 6 & 7 & 8 & \\
\hline $\begin{array}{l}\text { Obstacle } \\
\text { status }\end{array}$ & $\begin{array}{l}\text { Orientation } \\
\text { of robot }\end{array}$ & $\begin{array}{l}\text { Detection } \\
\text { value }\end{array}$ & No use \\
\hline
\end{tabular}

platform. The reflective IR sensors of the mobile robot detect cross point of the experimental platform. The mobile robot uses the compass module and the encoder of DC servomotors to compute the orientation and displacement of the mobile robot. The core of the wireless RF module is microprocessor (AT89C2051), and communicates with the controller via series interface (RS232). The communication protocol of the system contain one start byte, eight data bytes and one check byte, listed in Table 3.1.

The start byte trigs the wireless RF module to receive the signals from the transmitter. The data bytes contain ID code ( 1 byte), robot code (1 byte), and location and orientation data bytes. The ID code decides the transmitting direction between of the supervised computer and mobile robots. The robot code selects the mobile robot number. The control command protocol of the supervised computer is listed in Table.3.2. The mobile robot receives the command to discriminate the robot ID code to be right, moves to the target point step by step, and transmits the environment status to the supervised computer on real-time. The communication protocol of the feedback data from the mobile robot is listed in Table 3.3. The byte 5 represents the status of the obstacles in the experimental platform. The supervised computer receives the feedback data, knows the location of the mobile robot and the obstacles, programs the new trajectory to avoid obstacles for the mobile robot moving to the target floor, and receives the event signal from the event location to deal with the dangerous status.

Table 3.1: Communication Protocol

\begin{tabular}{|l|l|l|l|}
\hline Byte number & 0 & $1-8 \quad 9$ \\
\hline Definition & $\begin{array}{l}\text { Start } \\
\text { byte }\end{array}$ & $\begin{array}{l}\text { Data bytes (ID, } \\
\text { sensor, command, } \\
\text { robot, data) }\end{array}$ & $\begin{array}{l}\text { Check } \\
\text { sum }\end{array}$ \\
\hline
\end{tabular}

The control structure of each team robot system uses multilevel control structure to combine the merits of centralized control and decentralized control, shown in Fig. 3.1. The mobile robot communicates with the others of the team robot system and supervised computer via wireless RF interface and knows the location of each mobile robot. The mobile robot knows the command from the supervised computer or other mobile robot by ID code and robot ID code.

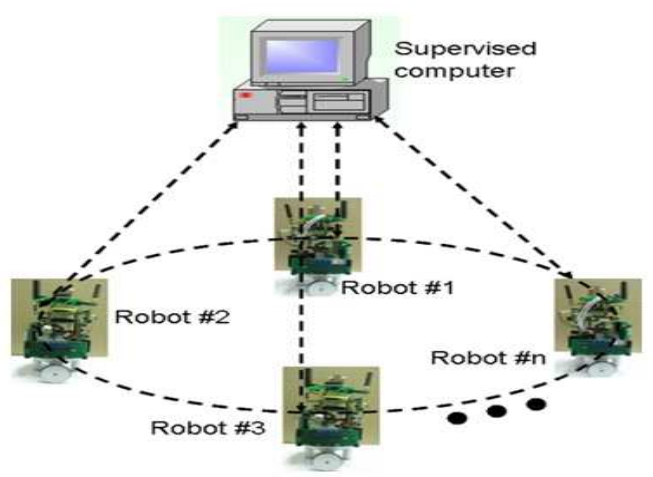

Fig. 3.1: Control Structure of Each Team Robot System

\section{Algorithm Analyze}

The Dempster-Shafter Evidence Theory is great utility in multisensor image segmentation to extend the application field for event detection of the security system. The development of Dempster-Shafter Evidence Theory began in the 1960s when Dempster $[9,10]$ developed the mathematical foundations of a two-value uncertainty mapping, upper and lower uncertainty measures, between 
two space. A result of this work is dempster's rule of combination, which operates on belief or mass functions as Baye's rule dose on probability functions.

A brief overview of the Dempster-Shafter Evidence Theory is provided as follows. Let $\phi$ be empty set and $\theta$ represents the set of hypotheses. The set of propositions $\left\{A_{j} \mid A_{j} \in 2^{\theta}\right\}$ for which a sensor is able to provide direct information, called "focal element". For each sensor $S_{i}$, the function:

$$
m_{i}:\left\{A_{j} \mid A_{j} \in 2^{\theta}\right\} \rightarrow[0,1]
$$

Termed a "basic probability assignment" maps a unit of probability mass or belief across the focal element of $S_{i}$ subject to the condition

$$
\begin{gathered}
m(\theta)=0 \\
\sum_{A_{j} \in 2^{\theta}} m_{i}\left(A_{j}\right)=1
\end{gathered}
$$

From this basic belief assignment $m$, a "belief" or "support" function is defined for $S_{i}$ as

$$
\operatorname{bel}_{i}(A)=\sum_{A_{j} \subseteq A} m_{j}\left(A_{j}\right)
$$

In a similar manner, "doubt" function, "plausibility" function, and "uncertainty" function are defined as [11]

$$
\begin{gathered}
d b t_{i}(A)=b e l_{i}\left(A^{c}\right) \\
p l s_{i}(A)=1-d b t_{i}(A) \\
u_{i}(A)=p l s_{i}(A)-b e l_{i}(A)
\end{gathered}
$$

The interval $[\operatorname{bel}(A), p l s(A)]$ is termed a "belief interval". "Dempster's rule of combination" is used to fuse the proposition $X$ and $Y$ from the two sensors $S_{i}$ and $S j$

$$
m_{i, j}(A)=\frac{\sum_{X \cap Y=A} m_{i}(X) m_{j}(Y)}{1-\sum_{X \cap Y=\phi} m_{i}(X) m_{j}(Y)}
$$

Where $A \neq \phi$, and where $m_{i, j}$ is the orthogonal sum $m_{i} \oplus m_{j}$, and $X, Y \in 2^{\theta}$. In addition, Dempster-Shafer Theory allows the fusion of several sources using the Dempster's combination operator. It is defined as:

$$
m(A)=m_{1}(A) \oplus \cdots \oplus m_{M}(A)
$$

\section{Experimental Results}

In the intelligent security system, we use three mobile robots as the fire detection team robot system to detect the fire source on the experimental platform. The mobile robot 1 of the fire detection team detects the fire source, a candle. The mobile robot 1 transmits the detection results to the supervised computer via wireless RF interface. The label of the mobile robot 1 displays red. The experimental results are shown in Fig.5.1 (a). The scenario of the fire experiment is shown in the right side.

Then the mobile robot 2 receives the event signal and moves on the platform to detect fire source, too. Robot 2 transmits the event signal to the supervised computer. The label of Robot 2 is red. The belief value is 0.7278 that is computed on the supervised computer using Dempster-Shafter Evidence Theory to be smaller than the threshold value. The label of the fire detection event is green. The experimental results are shown in Fig.5.1 (b). The mobile robot 3 moves to the fire event location, detects fire source, and transmits the event signal to the supervised computer. The belief value is 0.9617 over the threshold value using Dempster-Shafter Evidence Theory. The experimental results are shown in Fig.5.1 (c). In the condition, the supervised computer must alarm to users and transmits the fire event to users via variety interface to be shown on the right side of Fig.5.1 (c).

In the other case, we use three mobile robots to detect the gas leakage on the experimental platform, too. We use one lighter to provide gas on mobile robots 1 and 2 . They transmit the detection results to the supervised computer via wireless $\mathrm{RF}$ interface. The labels of the two mobile robots (1 and 2) are red. The experimental results are shown in Fig. 5.2 (a). The belief value is 0.8399 that is computed on the supervised computer using Dempster-Shafter Evidence Theory to be smaller than the threshold value. The label of the detection event is green. The mobile robot 3 detects gas leakage and transmits the event signal to the supervised computer. The belief value is 0.9709 over the threshold value using Dempster-Shafter Evidence Theory. The experimental results are shown in Fig. 5.2 (b). We set the probability values to be the same for each sensor of the mobile robots. The belief value of the gas leakage detection is calculated using Dempster-Shafter Evidence Theory, listed in Fig. 5.2 (c).

\section{Conclusions}

We have presented a multiple mobile robots based intelligent security system to be applied in intelligent home. The controller of the security modules and active modules (mobile robots) is HOLTEK microchip. We use Dempster-Shafter Evidence Theory to enhance the detection accuracy for the team based mobile robot system. Mobile robots can transmit real-time event signals to the supervised computer via wireless RF interface. In the paper, we use fire and gas leakage events to implement the function of the multiple mobile robots based security system for intelligent home. The experimental results are very nice to double check the event occurrence. In the future, we want to integrate passive security detection modules with the team robot system, use multisensory fusion algorithm to enhance the 


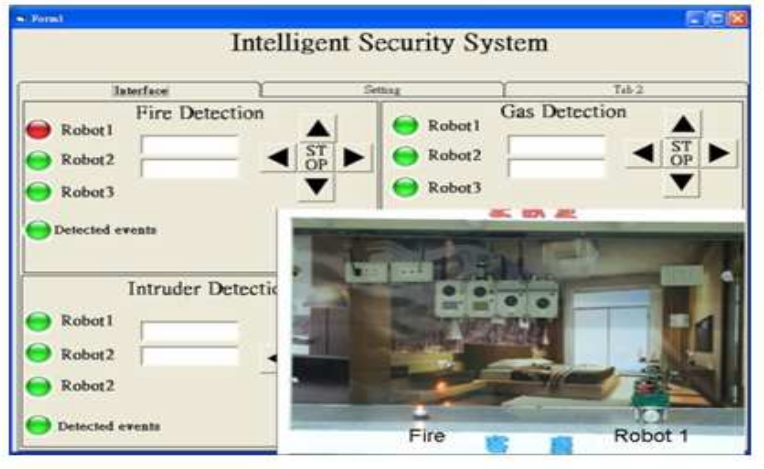

(a) Robot 1 detects fire source.

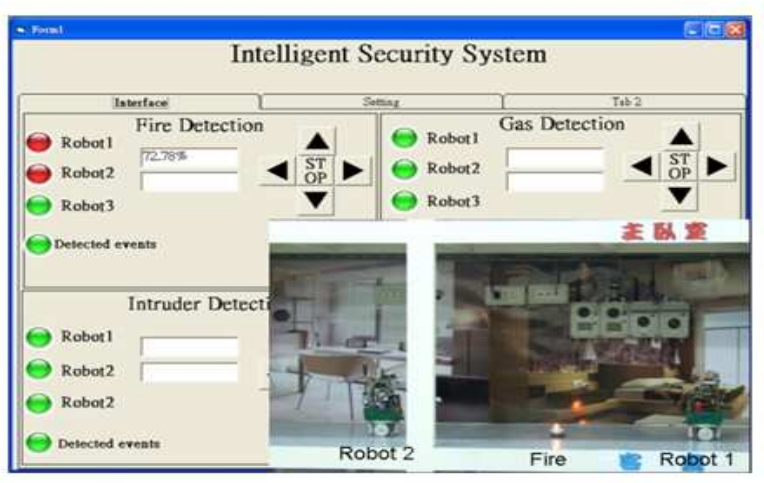

(b) Robot 1 and 2 detect fire source.

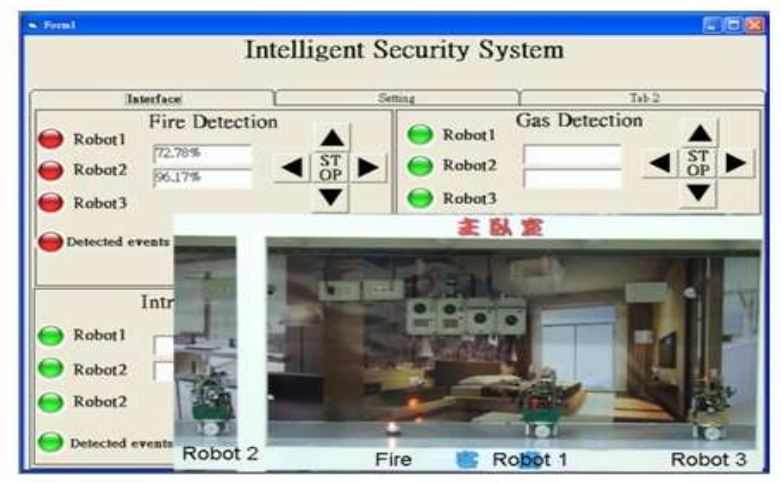

(c) Robot 1, 2 and 3 detect fire source.

Fig. 5.1: Experimental Results for Fire Source Detection

precision, and develop the remote supervised level to connect with the security system via Internet.

\section{Acknowledgements}

This work was supported by the project "Development of An Education Robot", under National Science Council of Taiwan, (NSC 99-2622-E-224-012-CC3).

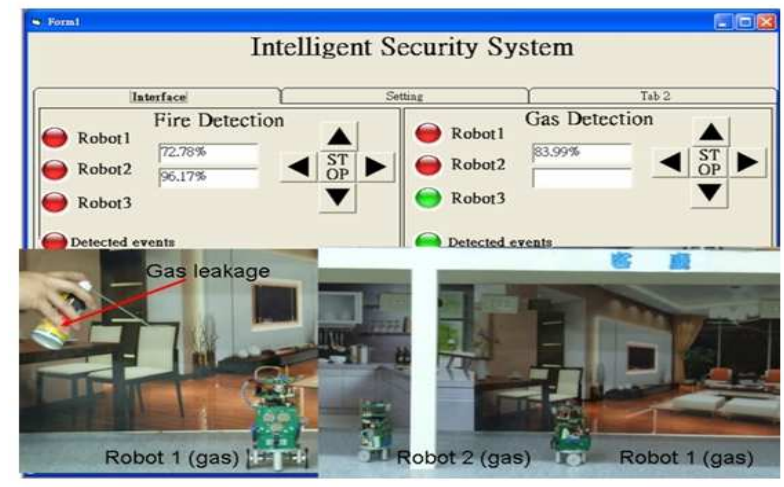

(a) Robot 1 and 2 detect gas leakage.

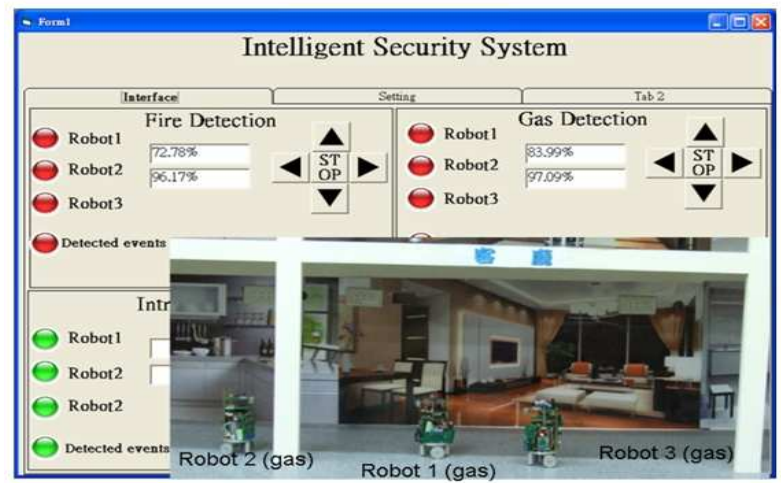

(b) Robot 1, 2 and 3 detect gas leakage.

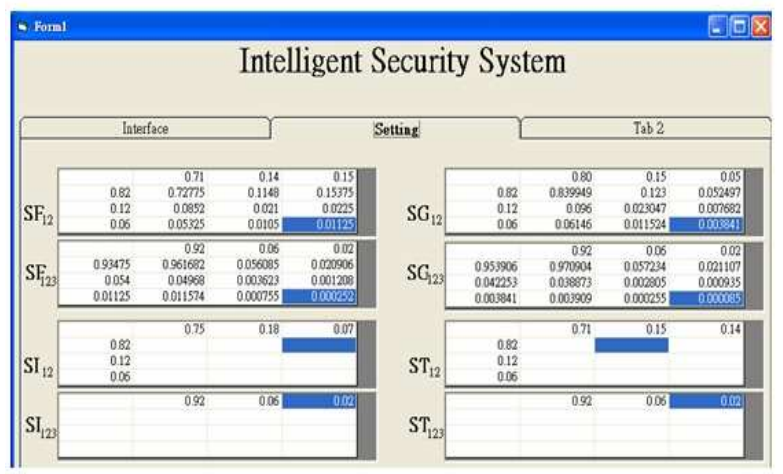

(c) Belief values

Fig. 5.2: Experimental Results for Fire Source Detection

\section{References}

[1] M. Azegami and H. Fujiyoshi, A systematic Approach to Intelligent Building Design, IEEE Communications Magazine (1993) 46-48.

[2] A. Kujuro and H. Yasuda, Systems Evolution in Intelligent Building, IEEE Communication Magazine (1993) 22-26.

[3] M. R. Finley and J. A. Karajura, Survey of Intelligent Building Concepts, IEEE Communication Magazine (1991) $18-20$. 
[4] W. Y. Chung and L. C. Fu, A Flexible, Hierarchical and Distributed Control Kernel Architecture for Rapid Resource Integration of Intelligent System, IEEE International Conference on Robotics Automation (2001) 1981-1987.

[5] Y. Shimosasa and J. Kanemoto, Some Results of The Test Operation of a Security Service System With Autonomous Guard Robot, IEEE International Conference on Industrial Electronic, Control, and Instrumentation (2010) 405-409.

[6] Y. Shimosasa and J. Kanemoto, Security Service System Using Autonomous placeMobile Robot, IEEE International Conference on System, Man, and Cybernetics (1999) 825829.

[7] R. Alami, S. Fleury, M. Herrb, F. Ingrand and F. Robert, Multi-robot cooperation in the MARTHA project, IEEE Robotics \& Automation Magazine, March (1998) 36-47.

[8] S. H. Chia and K. L. Su, Multi-level Multi-sensor Based Security System for Intelligent Home, International Symposium on Artificial Life and Robotics (AROB 15th), Beppu, Japan, Feb. 4-6 (2010) 379-382.

[9] G. A. Shafer, Mathemational Theory of Evidence, Princeton University Press, CityplacePrinceton, StateNJ, (1967).

[10] place E. Lefevre and O Colot, A Classification Method Based On the Dempster-Shafer's theory and information criteria, ISIF, (1967) 1179-7786.

[11] M. A. Abidi and R. C. Gonzalez, Data Fusion in Robotics and Machine Intelligence, Academic Press, Inc. (1992).

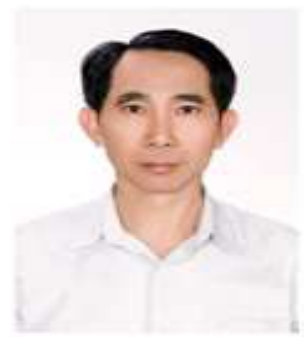

Song-Hiang

Chia

received the B. Sc. Degree in Electrical Engineering from the National Cheng Kung University, Tainan, Taiwan, R.O.C. in 1982. Now he is the Ph.D. candidate in Electrical Engineering at National Yunlin University of Science \& Technology, Taiwan, R.O.C. Since 1982, he has been at Wu-Feng Institute of Technology, Chiayi, Taiwan, teaching the subjects of Instrument Electronic, Electric Circuits, and Sensor and Transformer. Currently, he is a lecturer of Electronic Engineering at Wu-Feng University. His current research interests are in the areas of Control System and Module-Based Intelligent Mobile Robot Applications.

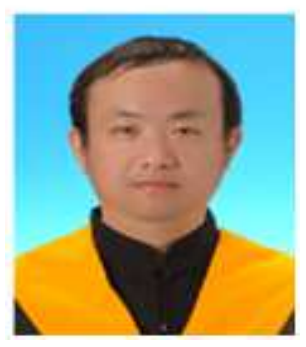

Jr-hung
Guo received the M. S. degree in Graduate School of OptoMechatronics and Materials, Wu-Feng Institute of Technology, Taiwan, R. O. C., and studies the Ph. D. program in Graduate School of Engineering Science and Technology, National Yunlin University of Science \& Technology, Taiwan. His research interests include microprocessor, artificial intelligence, and robotics.

$\mathbf{L i}$

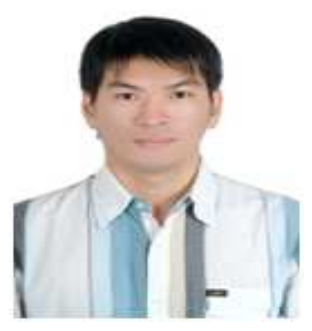

Bo Yi got a bachelor's degree at Kun Shan University and studies in Graduate School of Engineering Science and Technology, National Yunlin University of Science \& Technology, Douliou, Yunlin, Taiwan. His research interests include speech recognition and robotics.

Kuo Lan Su received the B. S. and M. S. degrees in Automatic Control Engineering from Feng-Chia University, Taichung, Taiwan, R. O. C., and received the PH. D. degree in Electrical Engineering at National Chung-Cheng University, Chia-Yi,Taiwan, R. O. C.. He is currently an associate professor in the Department of Electrical Engineering, National Yunlin University of Science \& Technology. His research interests include multisensor fusion and robotics. 